\title{
Factores asociados a seropositividad para SARS-CoV-2 en pacientes atendidos en un hospital de zona altoandina peruana
}

\section{Factors associated with seropositivity for SARS-CoV-2 in patients}

$\mathrm{DOI}$

https://doi.org/10.35434/rcmhnaaa.2021.14Sup1.1140

\section{RESUMEN}

Introducción: El COVID 19 es una enfermedad infecciosa causada por el SARS-CoV-2 que ha afectado en gran escala al Perú. No están documentados los potenciales factores clínicos y/o epidemiológicos que están relacionados a la positividad de SARS-CoV-2 en población altoandina. Objetivo: Determinar los factores asociados a positividad de SARS-Cov-2 en personas que viven en Ancash. Material y métodos: Estudio transversal analítico. Se utilizaron los registros de pacientes atendidos por sospecha de COVID-19 en un hospital público de Huaraz en marzo-mayo 2020. Se estimaron razones de prevalencia (RP) con intervalos de confianza. En el análisis de regresión simple, se estimaron razones de prevalencia e intervalos de confianza al 95\%, utilizando familia de distribución Poisson, función de enlace log y varianza robusta Resultados: De 903 pacientes, 13,7\% pacientes resultaron positivos a SARS-CoV-2. En la regresión simple, resultó que el sexo masculino $(R P=2,98)$, presentar tos $(R P=2,27)$, dificultad respiratoria $(R P=2,97)$, diarrea $(R P=2,69)$, malestar general $(R P=1,82)$, odinofagia $(R P=1,69)$ se asociaron positivamente a tener prueba SARS-CoV-2 positiva. En la regresión múltiple se mantuvo la asociación en cuatro características: ser varón $(\mathrm{RP}=2,7)$, presentar tos $(\mathrm{RP}=1,45)$, dificultad respiratoria $(\mathrm{RP}=2,15)$ y diarrea $(\mathrm{RP}=1,89)$. Conclusión: En zona altoandina, los casos positivos en su mayoría presentan síntomas típicos. Los factores asociados a la positividad fueron el sexo masculino y la presencia de dificultad tos, respiratoria y diarrea.

Palabras Clave: SARS-CoV-2; epidemiología; factores de riesgo; Perú (Fuente: DeCSBIREME).

\section{ABSTRACT}

Background: COVID 19 is an infectious disease caused by SARS-CoV-2 that has affected Peru on a large scale. Potential clinical and/or epidemiological factors that are related to the positivity of SARS-CoV-2 in the high Andean population are not documented. Objective: To determine factors associated with positivity of SARS-CoV-2 in people living in Ancash. Material and methods: Analytical transversal study. We used the records of patients treated for suspected COVID-19 in a public hospital in Huaraz in March-May 2020. Prevalence ratios (PR) with confidence intervals were estimated. In simple regression analysis, prevalence ratios and $95 \%$ confidence intervals were estimated, using Poisson distribution family, log-link function and robust variance. Results: Out of 903 patients, $13.7 \%$ tested positive for SARS-CoV-2. In simple regression, male ( $R P=2.98)$, cough $(R P=2.27)$, respiratory distress $(R P=2.97)$, diarrhea $(R P=2.69)$, general malaise $(R P=1.82)$, odynophagia $(\mathrm{RP}=1.69)$ were positively associated with having a positive SARS-CoV-2 test. In the multiple regressions, the association was maintained in four characteristics: being male $(R P=2.7)$, having a cough $(R P=1.45)$, respiratory distress $(R P=2.15)$, and diarrhea $(R P=1.89)$. Conclusion: In high Andean areas, most positive cases present typical symptoms. The factors associated with positivity were male sex and the presence of cough, respiratory difficulty and diarrhea.

Keywords: SARS-CoV-2; Seroepidemiology; risk factors; Peru. (Source: DeCS-BIREME).
FILIACIÓN

1. Instituto de Investigación en Ciencias Biomédicas de la Universidad Ricardo Palma, Lima, Perú.

2. Universidad Nacional de Trujillo, Trujillo, Perú.

2. Hospital Víctor Ramos Guardia, Huaraz, Perú.

4. Facultad de Medicina, Universidad Nacional Federico Villareal, Lima, Perú.

5. Universidad San Ignacio de Loyola, Lima, Perú.

. Universidad Norbert Wiener, Lima, Perú.

a. Médico.

\section{ORCID}

1. Victor J Vera-Ponce / 0000-0003-4075-9049

2. Paul Mendez-Aguilar $/$ 0000-0003-2140-4138

3. C. Ichiro Peralta / 0000-0003-0971-8708

4. Virgilio E Failoc-Rojas / 0000-0003-2992-9342

5. Mario J Valladares-Garrido / 0000-0003-0839-2419

\section{CORRESPONDENCIA}

Virgilio E Failoc-Rojas, M.D, MSc (c). Universidad San Ignacio de Loyola.

Dirección: Av La Fontana 505, La Molina, Lima, Peru.

Telefono: +51948845837

EMAIL

virgiliofr@gmail.com

CONFLICTOS DE INTERÉS

Los autores declaran no tener conflictos de intereses.

FINANCIAMIENTO

Autofinanciamiento.

CONTRIBUCIÓN DEAUTORÍA

VJVP y PMA participaron en la concepción y diseño del artículo, recolección de resultados. CIP, VEFR, MJVG participaron en el análisis e interpretación de datos. Todos los autores participaron en la redacción del artículo, revisión crítica del artículo, aprobación la redacción del a

\section{REVISIÓN DE PARES}

A los profesionales médicos del Hospital Víctor Ramos Guardia, Huaraz-Perú.

Recibido: $17 / 05 / 2021$

Aceptado: 06/09/2021

\section{COMO CITAR}

Vera-Ponce, V., Mendez-Aguilar, P., Ichiro-Peralta, C., Failoc-Rojas, V., \& Valladares-Garrido, M. (2021). Factores asociados a seropositividad para SARS-CoV-2 en pacientes atendidos en un hospital de zona altoandina peruana. Revista Del Cuerpo Médico Hospital Nacional Almanzor Aguinaga Asenjo, 14(Sup1), 8 - 12. https://doi.org/10.35434/rcmhnaaa.2021.14Sup1.1140

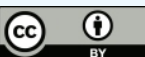

Esta obra está bajo una Licencia Creative Commons Atribución 4.0 Internacional.

Versión Impresa: ISSN: 2225-5109

Versión Electrónica: ISSN: 2227-4731

Cross Ref. DOI: 10.35434/rcmhnaaa

OJS: https://cmhnaaa.org.pe/ojs 


\section{INTRODUCCIÓN}

El COVID 19 es una enfermedad infecciosa causada por el SARS-CoV-2 que apareció en la ciudad de Wuhan, China, a finales del $2019^{(1)}$. Las personas con esta enfermedad manifiestan una variabilidad de síntomas, caracterizado por la tos, fiebre, odinofagia y disnea ${ }^{(2)}$. El diagnóstico por COVID-19 es a través de la prueba rápida y/o prueba molecular ${ }^{(3)}$. No obstante, frente a la escasez de las mismos, se utilizan los criterios clínicos para la sospecha; más aún cuando se trata de la tomar la decisión de aislar a un paciente de manera ambulatoria ${ }^{(4)}$. Frente a esa problemática, es importante conocer las características clínicas que acompañan con mayor frecuencia a esta enfermedad.

Existen estudios relacionados al COVID-19 en el país, pero debemos considerar que estos no se han centrado explícitamente en valorar los factores relacionados a positividad de SARS-CoV- $2^{(5-7)}$. A su vez, la mayoría de estudios en el país trabaja con pacientes hospitalizados ${ }^{(5,6,8)}$, existen limitaciones encontradas, como el poco tamaño de muestra ${ }^{(5)}$, haberse realizado en pacientes que dieron positivo y se encontraban a la espera de ser atendidos en el servicio de emergencia ${ }^{(8)}$. Las adaptaciones fisiológicas debido a la altitud podrían proteger del impacto severo de la infección aguda por este virus ${ }^{(9)}$, el cual podría diferir en las características que presente un paciente infectado. Sin embargo, tampoco aún no están documentados los potenciales factores clínicos y/o epidemiológicos que están relacionados a la positividad de SARS-CoV-2, mucho menos en zonas altoandinas peruanas.

En este contexto, se necesita elaborar futuras decisiones a nivel de salud, orientados a identificar de forma precoz los potenciales factores relacionados a positividad de esta enfermedad. A su vez, conocer si las características clínicas de los sujetos seropositivos a SARS-CoV-2 residentes en altura difieren con el resto de la población peruana. Por eso mismo, el objetivo de la investigación es determinar los factores asociados a positividad de SARS-Cov-2 en pacientes atendidos en un hospital público de Huaraz, Perú.

\section{MATERIAL Y MÉTODOS}

\section{Diseño del estudio}

Estudio transversal analítico cuyo propósito fue identificar los factores asociados a positividad para SARS-CoV-2 en pacientes atendidos en un hospital público de Huaraz, Perú durante el periodo de emergencia sanitaria por COVID-19.

\section{Población y muestra}

La población estuvo constituida por pacientes atendidos por sospecha de COVID-19 en el Hospital Víctor Ramos Guardia, Huaraz-Perú durante el periodo 13 de marzo al 9 de mayo del 2020. La muestra estuvo conformada por 794 pacientes con sospecha de COVID-19. Se incluyeron a los pacientes a quienes se les realizó la ficha de investigación epidemiológica para COVID-19 del Ministerio de Salud. Se excluyeron a pacientes referidos de otra sede hospitalaria dado que ya contaban con la notificación epidemiológica realizada en su respectivo establecimiento de salud.

\section{Procedimientos de estudio}

Primero, un investigador del estudio, quien desempeña actividades de vigilancia epidemiológica en la Oficina de Epidemiología del hospital, fue el encargado de realizar el proceso de control de calidad de las fichas epidemiológicas previo a la notificación en el Sistema de Notificación del Ministerio de Salud (NotiWeb-MINSA). Luego, las fichas fueron debidamente notificadas en dicho sistema de ingreso de datos. Finalmente, se exportó la base de datos desde el NotiWeb en formato Microsoft Excel 2016 para realizar el respectivo procesamiento de la información.

\section{Instrumentos y variables}

El instrumento del estudio estuvo constituido por el formato de la ficha de investigación clínica epidemiológica de COVID19 del MINSA. Esta ficha epidemiológica está constituida por 1) datos generales de notificación y del paciente (fecha de notificación, clasificación del caso, sexo, edad, departamento, ocupación), 2) cuadro clínico (fecha de inicio de síntomas, reporte de aislamiento, reporte de tos, dolor de garganta, congestión nasal, dificultad respiratoria, fiebre/escalofrío, diarrea, malestar general, náuseas/vómitos, cefalea, irritabilidad/confusión, dolor muscular/pecho/abdominal/articulaciones), 3 ) comorbilidades (embarazo, enfermedad cardiovascular, diabetes, enfermedad hepática, enfermedad crónica neurológica/neuromuscular, inmunodeficiencia, enfermedad renal, daño hepático, enfermedad pulmonar crónica y cáncer), 4) historia previa de viaje y exposición 14 días previos al inicio de síntomas y 5) hallazgos de laboratorio (fecha de toma de muestra, tipo de muestra, resultado).

La variable desenlace fue positividad para SARS-CoV-2, definida operacionalmente como la detección del virus SARSCoV-2 en la prueba diagnóstica (serológica/molecular) realizada a los pacientes con sospecha de COVID-19 atendidos en el hospital altoandino peruano. Las categorías de medición fueron reactiva/no reactiva.

Las variables de exposición fueron las características clínicoepidemiológicas: edad en años, sexo (masculino, femenino), reporte de síntomas según la descripción de la ficha epidemiológica realizada previamente (no, si).

\section{Análisis estadístico}

El análisis estadístico fue realizado en el programa STATA v.16.1 (StataCorp LP, College Station, TX, USA).

Se describieron medidas de tendencia central y de dispersión para las variables numéricas, previa evaluación de supuesto de distribución normal. En variables categóricas, se reportaron frecuencias absolutas y relativas.

En el análisis bivariado de variables categóricas, se utilizó la prueba de chi cuadrado para explorar asociación entre factores clínicos-epidemiológicos y positividad para SARSCoV-2, previa evaluación de supuesto de frecuencias esperadas. Para variables numéricas, se utilizó la prueba t de student para muestras independientes, caso contrario la prueba de U de Mann Whitney.

En el análisis de regresión simple, se estimaron razones de prevalencia e intervalos de confianza al 95\%, utilizando familia de distribución Poisson, función de enlace log y varianza robusta. Para el modelo múltiple, se construyó un modelo parsimónico realizando proceso de anidamiento 
entre las variables que resultaron aportar significativamente al modelo, utilizando la prueba de Likelihood-ratio test. Aspectos Éticos.

Esta investigación ha sido aprobada por el Comité de Ética del Hospital Víctor Ramos Guardia, Huaraz-Perú. Se utilizaron códigos para mantener la confidencialidad de los datos de los pacientes con sospecha de COVID-19 elegibles para este estudio. Se respetaron los principios éticos de la Declaración de Helsinki. El proyecto de investigación fue registrado en el repositorio de proyectos de investigación en salud del Instituto Nacional de Salud-Perú (PRISA).

\section{RESULTADOS}

Se recolectó información de 794 pacientes, de ellos 109 $(13,8 \%)$ dieron positivo. La mediana de edad fue similar en pacientes con diagnóstico positivo y la población general, con rangos de edad de 6 meses a 89 años. El tiempo de síntomas fue mayor en quienes dieron positivo frente a la población general (7 días y 4 días respectivamente). De los que tenían infección, 33,9\% eran asintomáticos, y de los que presentaron síntomas la más frecuente fue tos y odinofagia $(49,5 \%$ y $44,0 \%$ respectivamente). Se encontró pocas comorbilidades en la población estudiada, la más frecuente fue la obesidad (3\%). El 38,3\% fue personal de salud. Más información en la Tabla 1.

Tabla 1. Características clínico epidemiológicas de pacientes con sospecha de COVID-19 en zona altoandina en Perú, marzo-mayo 2020.

\begin{tabular}{|c|c|c|c|c|}
\hline \multirow{2}{*}{ Características } & \multicolumn{2}{|c|}{ Positivos } & \multicolumn{2}{|c|}{ Total } \\
\hline & $(\mathrm{N}=109)$ & $\%^{* *}$ & $(\mathrm{~N}=794)$ & $\%$ \\
\hline \multicolumn{5}{|l|}{ Características } \\
\hline Edad (años) * & 41,6 & 17,8 & 39,5 & 15,4 \\
\hline Tiempo de síntomas (Días) \pm & 7 & 9 & 4 & 6 \\
\hline \multicolumn{5}{|l|}{ Sexo } \\
\hline Masculino & 82 & 75,2 & 401 & 50,5 \\
\hline Femenino & 27 & 24,8 & 393 & 49,5 \\
\hline \multicolumn{5}{|l|}{ Procedencia } \\
\hline Ancash & 107 & 98,2 & 791 & 99,6 \\
\hline Otro & 2 & 1,8 & 3 & 0,4 \\
\hline \multicolumn{5}{|l|}{ Síntomas (Sí) } \\
\hline Fiebre/escalofrío & 34 & 31,2 & 147 & 18,5 \\
\hline Malestar general & 37 & 33,9 & 175 & 22 \\
\hline Tos & 54 & 49,5 & 243 & 30,6 \\
\hline Odinofagia & 48 & 44 & 252 & 31,7 \\
\hline Congestión nasal & 26 & 23,9 & 126 & 15,9 \\
\hline Dificultad respiratoria & 35 & 32,1 & 109 & 13,7 \\
\hline Diarrea & 13 & 11,9 & 38 & 4,8 \\
\hline Nauseas & 10 & 9,2 & 31 & 3,9 \\
\hline Cefalea & 13 & 11,9 & 95 & 12 \\
\hline Asintomáticos & 37 & 33,9 & 377 & 47,5 \\
\hline \multicolumn{5}{|l|}{ Factor de riesgo } \\
\hline Enfermedad cardiovascular & 2 & 1,8 & 22 & 2,8 \\
\hline Diabetes Mellitus 2 & 2 & 1,8 & 17 & 2,5 \\
\hline Obesidad & 4 & 3,7 & 24 & 3 \\
\hline Neoplasia & 1 & 0,9 & 1 & 0,1 \\
\hline VIH & 1 & 0,9 & 1 & 0,1 \\
\hline
\end{tabular}

* Se presenta resultados en media (desviación estándar). I Se presenta resultados en mediana (rango interquartilico). ${ }^{* *}$ Porcentajes obtenidos solo de pacientes positivos a SARS-CoV-2.
Hubo diferencias estadísticamente significativas en el grupo por sexo (varones y mujeres tenían frecuencias de tener positividad en $20,5 \%$ y $6,9 \%$ respectivamente, $p<0,001$ ), así como el tiempo de síntomas en días fue mayor en quienes dieron positivo que negativo $(\mathrm{p}<0,001)$. Los pacientes que reportaban fiebre, malestar general, tos, odinofagia, congestión nasal disnea o diarrea tenían más probabilidad de tener seropositividad a SARS-CoV-2. Más información en la tabla 2.

Tabla 2. Diferencias clínico epidemiológico según coinfección de COVID-19 en zona altoandina en Perú, marzomayo 2020.

\begin{tabular}{|c|c|c|c|}
\hline & Positivo & Negativo & Valor-p \\
\hline \multicolumn{4}{|l|}{ Características } \\
\hline Edad (años) * & $41,61(14,8)$ & $39,23(15,4)$ & 0,139 \\
\hline Tiempo de síntomas (Días) \pm & $7(9)$ & $3(6)$ & $<0,001$ \\
\hline \multicolumn{4}{|l|}{ Sexo** } \\
\hline Masculino & $82(20,5)$ & $319(79,5)$ & $<0,001$ \\
\hline Femenino & $27(6,9)$ & $366(93,1)$ & \\
\hline \multicolumn{4}{|l|}{ Síntomas** } \\
\hline Fiebre/escalofrío(Sí) & $34(23,1)$ & $113(76,9)$ & $<0,001$ \\
\hline Fiebre/escalofrío(No) & $75(11,6)$ & $572(88,41)$ & \\
\hline Malestar general (Sí) & $37(21,1)$ & $138(78,9)$ & 0,001 \\
\hline Malestar general (No) & $72(11,6)$ & $547(88,4)$ & \\
\hline Tos $(\mathrm{Si})$ & $54(22,2)$ & $189(77,8)$ & $<0,001$ \\
\hline Tos (No) & $55(10,0)$ & $496(90,0)$ & \\
\hline Odinofagia (Si) & $48(19,1)$ & $204(80,9)$ & 0,003 \\
\hline Odinofagia (No) & $61(11,3)$ & $481(88,8)$ & \\
\hline Congestión nasal (Si) & $26(20,6)$ & $100(79,4)$ & 0,014 \\
\hline Congestión nasal (No) & $83(12,4)$ & $585(87,6)$ & \\
\hline Dificultad respiratoria $(\mathrm{Si})$ & $35(32,1)$ & $74(67,9)$ & $<0,001$ \\
\hline Dificultad respiratoria (No) & $74(10,8)$ & $611(89,2)$ & \\
\hline Diarrea (Si) & $13(34,2)$ & $25(65,8)$ & $<0,001$ \\
\hline Diarrea (No) & $96(12,7)$ & $660(87,3)$ & \\
\hline Nauseas (Si) & $10(32,3)$ & $21(67,7)$ & 0,002 \\
\hline Nauseas (No) & $99(13,0)$ & $664(87,0)$ & \\
\hline Cefalea (Si) & $13(13,7)$ & $84(86,3)$ & 0,989 \\
\hline Cefalea (No) & $96(13,7)$ & $603(86,3)$ & \\
\hline Asintomático (Si) & $37(9,8)$ & $340(90,2)$ & 0,002 \\
\hline Asintomático (No) & $72(17,3)$ & $345(82,7)$ & \\
\hline
\end{tabular}

Withney. ** Se usó prueba de chi2.

En la regresión simple, ser de sexo masculino (RP:2,98) y tener síntomas como tos (RP: 2,27), disnea (RP: 2,97), diarrea (RP:2,69), fiebre (RP: 1,99), malestar general (RP:1,82), odinofagia (RP: 1,69), congestión nasal $(R P: 1,66)$ y nauseas (RP:2,49) se asociaron con seropositividad para SARS-CoV-2. En la regresión múltiple, solo ser de sexo masculino, tener tos, disnea y diarrea se asoció positivamente con seropositividad. Ver la tabla 3. 
Tabla 3. Factores asociados a SARS-Cov-2 positivo en zona altoandina del Perú, marzo-mayo 2020.

\begin{tabular}{|c|c|c|c|c|c|c|}
\hline \multirow{2}{*}{ Características } & \multicolumn{3}{|c|}{ Modelo crudo* } & \multicolumn{3}{|c|}{ Modelo parsimónico** } \\
\hline & RPc & IC $95 \%$ & Valor-p & $\mathrm{RPa}$ & IC $95 \%$ & Valor-p \\
\hline \multicolumn{7}{|l|}{ Sexo } \\
\hline Masculino & 2,98 & $1,93-4,60$ & $<0,001$ & 2,7 & $1,79-4,06$ & $<0,001$ \\
\hline Femenino & Ref & & & Ref & & \\
\hline \multicolumn{7}{|l|}{ Síntomas } \\
\hline Tos (Si) & 2,27 & $1,53-3,24$ & $<0,001$ & 1,45 & $1,00-2,10$ & 0,053 \\
\hline Tos (No) & Ref & & & Ref & & \\
\hline Dificultad respiratoria $(\mathrm{Si})$ & 2,97 & $1,99-4,44$ & $<0,001$ & 2,15 & $1,49-3,11$ & $<0,001$ \\
\hline Dificultad respiratoria (No) & Ref & & & Ref & & \\
\hline Diarrea (Si) & 2,69 & $1,51-4,81$ & $<0,001$ & 1,89 & $1,17-3,04$ & 0,009 \\
\hline Diarrea (No) & Ref & & & Ref & & \\
\hline Fiebre/escalofrio(Sí) & 1,99 & $1,33-2,99$ & 0,001 & NA & & \\
\hline Fiebre/escalofrio(No) & Ref & & & & & \\
\hline Malestar general (Sí) & 1,82 & $1,22-2,70$ & 0,003 & NA & & \\
\hline Malestar general (No) & Ref & & & & & \\
\hline Odinofagia (Si) & 1,69 & $1,16-2,47$ & 0,006 & NA & & \\
\hline Odinofagia (No) & Ref & & & & & \\
\hline Congestion nasal (Si) & 1,66 & $1,07-2,58$ & 0,024 & NA & & \\
\hline Congestion nasal (No) & Ref & & & & & \\
\hline Nauseas (Si) & 2,49 & $1,30-4,76$ & 0,006 & NA & & \\
\hline Nauseas (No) & Ref & & & & & \\
\hline Cefalea (Si) & 0,99 & $0,56-1,78$ & 0,99 & NA & & \\
\hline Cefalea (No) & Ref & & & & & \\
\hline
\end{tabular}

*Se usó modelos lineales generalizados con familia poisson, enlace logaritmo para estimar Razones de Prevalencia de la exposición con seropositividad a SARS-CoV-2. ${ }^{* * *}$ Se usó
modelos lineales generalizados con familia poisson, enlace logaritmo mediante modelo marsimónico con forward selection $(p<0.2)$ para estimar Razones de Prevalencia de la exposición con seropositividad a SARS-COV-NA: No ingresaron al modelo parsimónico.

\section{DISCUSIÓN}

Este estudio da luces sobre las características clínicoepidemiológicas de pacientes sintomatológicos y confirmados de COVID-19 que residen en una zona de altitud geográfica en Perú. Nuestros resultados muestran que la prevalencia de positividad a SARS-CoV-2 fue de $13,7 \%$ y los factores asociados a esta positividad fueron el sexo masculino y la presencia de dificultad respiratoria y diarrea.

Considerando que parte de nuestra muestra incluyó a contactos, es esperable observar que nueve de cada diez sospechosos muestren negatividad a SARS-CoV-2, suponiendo además que muchos de ellos no tuvieron una exposición alta al virus. Además, observamos que la mayoría de los casos positivos tuvieron síntomas típicos, lo que sugiere que un importante número fue detectado posiblemente durante la trazabilidad de contactos.

Por otro lado, es interesante destacar que, en nuestro estudio, poco más de $90 \%$ de pacientes sintomáticos mostró negatividad a SARS-CoV-2. Suponemos que esto pudo deberse a una frecuencia elevada de falsos negativos (en parte por el mayor uso de pruebas serológicas) y, con menor probabilidad, a una infección de etiología viral distinta. Respecto al valor diagnóstico de las pruebas serológicas y moleculares, no sorprende encontrar resultados falsos negativos en fases sintomáticas iniciales ${ }^{(9,10)}$. Esto refuerza la recomendación de mantener a los casos sospechosos en aislamiento, así como de emplear prioritariamente las pruebas moleculares.

Se observó que la prevalencia de casos positivos fue mayor en aquellos que presentaron dificultad respiratoria y diarrea. Estos resultados extienden los hallazgos de estudios previos que abarcaron a población de altura ${ }^{(9,11,12)}$, donde se presentan datos heterogéneos y contradictorios sobre la sintomatología por COVID-19. En relación con estudios internacionales ${ }^{(13,14)}$ y peruanos ${ }^{(5)}$, es coherente que uno de los síntomas encontrados sea la dificultad respiratoria (además de la tos y fiebre, síntomas no incluidos en el modelo multivariable, pero entre los más frecuentes de nuestro estudio), sobre todo en fases moderadas o graves de la enfermedad. Sin embargo, la diarrea ha sido uno de los síntomas menos frecuentemente descritos en la literatura ${ }^{(13,14)}$. Asumiendo que pocos pacientes han reportado diarrea, es posible que la asociación hallada sea consecuencia de una limitación en la potencia estadística más que una diferencia intrínseca en nuestra población de estudio.

Varios factores intentan explicar el posible efecto de la altura sobre el SARS-CoV-2, así como sobre la susceptibilidad y progresión de la enfermedad ${ }^{(15)}$. Entre los más destacables se encuentran los factores ambientales (presencia de rayos UV y ozono) $^{(9,16)}$ y fisiológicos (hipoxia y menor expresión de receptores $\mathrm{ACE}-2)^{(9)}$.

Encontramos que los varones tuvieron una prevalencia 177\% mayor de ser positivos a SARS-CoV-2. Este hallazgo se condice con lo reportado previamente, en donde se identificaron que los hombres tienen un mayor riesgo de infección ${ }^{(17)}$, severidad y mortalidad por COVID-19 ${ }^{(18)}$. Múltiples hipótesis explican la mayor prevalencia de infección en varones, entre ellas los hábitos sociales como el tabaquismo, la percepción de búsqueda de atención médica y la existencia de comorbilidades $^{(19)}$. Por otro lado, desde una perspectiva biológica, se ha postulado una relación con la respuesta inmunológica, en la cual intervienen factores epigenéticos, cromosómicos y hormonales ${ }^{(20)}$. Estos determinan una mayor expresión de los receptores ACE-2 y TMPRSS2, así como una deficiente respuesta innata y adaptativa, las cuales predisponen a la susceptibilidad de infección y al incremento de las tasas de fatalidad en la población masculina.

Se recomienda el diseño de un estudio nacional de cohorte, con mayor tamaño de muestra, estratificado por altitud geográfica y gravedad de enfermedad, adicionando información radiológica y laboratorial, y usando más pruebas moleculares, para identificar adecuadamente las características clínicas de pacientes con sospecha de infección por SARS-CoV-2 en regiones de altura.

Este estudio destaca las características clínicoepidemiológicas de pacientes con sospecha de SARS-CoV-2 en una región geográfica de altura. Los factores asociados a la positividad serológica o molecular fueron el sexo masculino y la presencia de dificultad respiratoria y diarrea.

\section{REFERENCIAS BIBLIOGRÁFICAS}

1. Hassan SA, Sheikh FN, Jamal S, Ezeh JK, Akhtar A. Coronavirus (COVID-19): A Review of Clinical Features, Diagnosis, and Treatment. Cureus. 2020;12(3):e7355. doi: 10.7759 / cureus.7355

2. Wiersinga WJ, Rhodes A, Cheng AC, Peacock SJ, Prescott HC. Pathophysiology, Transmission, Diagnosis, and Treatment of Coronavirus Disease 2019 (COVID-19): A Review. Jama. 2020; 324 (8): 782-793. doi: 10.1001 / jama.2020.12839 
3. Iyer M, Jayaramayya K, Subramaniam MD, Lee SB, Dayem AA, Cho SG, et al. COVID-19: an update on diagnostic and therapeutic approaches. BMB reports. 2020;53(4):191-205. doi: 10.5483/BMBRep.2020.53.4.080

4. Rojas JPM, Loría MQ, Sánchez MG, Chinchilla AS. SARS CoV-2, manifestaciones clínicas y consideraciones en el abordaje diagnóstico de COVID-19. Revista Auspiciada por el Hospital Dr Rafael Ángel Calderón Guardia. [Internet] 2020 [citado el 18 de setiembre de

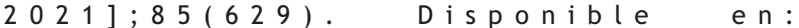
http://revistamedicacr.com/index.php/rmcr/article/view/287/26 $\underline{4}$

5. Acosta G, Escobar G, Bernaola G, Alfaro J, Taype W, Marcos C, et al. Caracterización de pacientes con COVID-19 grave atendidos en un hospital de referencia nacional del Perú. Revista Peruana de Medicina Experimental y Salud Pública. 2020;37(2). doi: 10.17843/rpmesp.2020.372.5437

6. Benites-Goñi H, Vargas-Carrillo E, Peña-Monge E, Taype-Rondan A, Arróspide-Mormontoy D, Castillo-Córdova M, et al. Características clínicas, manejo y mortalidad de pacientes hospitalizados con COVID19 en un hospital de referencia en Lima, Perú. Pre-print. [Internet] 2020. [citado el 18 de setiembre de 2021] Disponible en: https://preprints.scielo.org/index.php/scielo/preprint/download/ 905/1266/1325

7. Llaro-Sánchez MK, Gamarra-Villegas BE, Campos-Correa KE. Características clínico-epidemiológicas y análisis de sobrevida en fallecidos por COVID-19 atendidos en establecimientos de la Red Sabogal-Callao 2020. Horiz. Med. 2020;20(2). doi: 10.24265/horizmed.2020.v20n2.03

8. Valenzuela GV, Rodríguez-Morales AJ, Mamani R, Ayala R, Pérez K, Sarmiento $\mathrm{C}$, et al. Cardiovascular risk factors and evolution of patients attended with COVID-19 in a National Reference Hospital from Lima, Peru. 2020. 2021;5(3):195-200. doi: 10.35839/repis.5.3.1071

9. Arias-Reyes C, Zubieta-DeUrioste N, Poma-Machicao L, AliagaRaduan F, Carvajal-Rodriguez F, Dutschmann M, et al. Does the pathogenesis of SARS-CoV-2 virus decrease at high-altitude? Respiratory physiology \& neurobiology. 2020;277:103443. doi: 10.1016/j.resp.2020.103443.

10. Kong W, Wang Y, Hu J, Chughtai A, Pu H. Comparison of clinical and epidemiological characteristics of asymptomatic and symptomatic SARS-CoV-2 infection: A multi-center study in Sichuan Province, China. Travel medicine and infectious disease. 2020:101754. doi: 10.1016/j.tmaid.2020.101754.
11. Xi A, Zhuo M, Dai J, Ding Y, Ma X, Ma X, et al. Epidemiological and clinical characteristics of discharged patients infected with SARSCoV-2 on the Qinghai Plateau. Journal of medical virology. 2020;92(11):2528-2535. doi: 10.1002/jmv.26032.

12. Zeng J, Peng S, Lei Y, Huang J, Guo Y, Zhang X, et al. Clinical and Imaging features of COVID-19 Patients: Analysis of Data from HighAltitude Areas. The Journal of infection. 2020;80(6):e34-e6. doi: 10.1016 / j.jinf.2020.03.026.

13. Chen J, Lu H, Melino G, Boccia S, Piacentini M, Ricciardi W, et al. COVID-19 infection: the China and Italy perspectives. Cell death \& disease. [Internet] 2020 [citado el 18 de setiembre de 20021 ] ; 11 ( 6 ) : 438 . D i s p o n i ble e n: https: / / www.nature.com/articles/s41419-020-2603-0

14. Guan W-j, Ni Z-y, Hu Y, Liang W-h, Ou C-q, He J-x, et al. Clinical characteristics of coronavirus disease 2019 in China. New England journal of medicine. 2020;382(18):1708-20. doi: 10.1056 / NEJMoa2002032

15. Burtscher J, Burtscher M, Millet GP. Caution is needed on the effect of altitude on the pathogenesis of SAR-CoV-2 virus. Respiratory physiology \& neurobiology. 2020;279:103464. doi: 10.1016 / j.resp.2020.103464

16. Semple JL, Moore GWK. High levels of ambient ozone (03) may impact COVID-19 in high altitude mountain environments. Respiratory physiology \& neurobiology. 2020;280:103487. doi: 10.1016 / j.resp.2020.103487

17. Jehi L, Ji X, Milinovich A, Erzurum S, Rubin BP, Gordon S, et al. Individualizing Risk Prediction for Positive Coronavirus Disease 2019 Testing: Results from 11,672 Patients. Chest. 2020; 158(4):13641375. doi:10.1016 / j.chest.2020.05.580

18. Jin JM, Bai P, He W, Wu F, Liu XF, Han DM, et al. Gender Differences in Patients With COVID-19: Focus on Severity and Mortality. Frontiers in public health. 2020;8:152. doi:10.3389/fpubh.2020.00152

19. Meng Y, Wu P, Lu W, Liu K, Ma K, Huang L, et al. Sex-specific clinical characteristics and prognosis of coronavirus disease-19 infection in Wuhan, China: A retrospective study of 168 severe patients. PLoS pat hogens. 2020;16(4): e 1008520 . doi : 10.1371 /journal.ppat. 1008520

20. Scully EP, Haverfield J, Ursin RL, Tannenbaum C, Klein SL. Considering how biological sex impacts immune responses and COVID-19 outcomes. Nature reviews Immunology [Internet]. 2020 [Citado el 18 de setiembre de 2021];20(7):442-7. Disponible en: https://www.nature.com/articles/s41577-020-0348-8. 